\title{
MÖGLICH
}

\section{Subgruppenanalysen dürfen nicht dazu führen, Diabetikern ARNI vorzuenthalten!}

\author{
Im Mai wurden die neuen Leitlinien der Europäischen Kardiologengesellschaft ESC zu \\ Herzinsuffizienz (HI) vorgestellt [1]. Die Therapie chronisch herzinsuffizienter Patienten \\ mit eingeschränkter Pumpfunktion wurde mit Einführung der neuen Substanzklasse \\ der Angiotensinrezeptor-Neprilysin-Inhibitoren (ARNI) revolutioniert. Was gilt derzeit für \\ Betroffene mit Diabetes mellitus als Grund-/Begleiterkrankung?
}

Der Vertreter der ARNI Sacubitril/Valsartan (LCZ696) hemmt Neprilysin und somit die Degradation von natriuretischen sowie vasodilatierenden Peptiden und blockiert die durch den Angiotensin-I-Rezeptor vermittelte Wirkung. Geprüft wurde LCZ696 in der PARADIGM-HF-Studie. Der primäre kombinierte Endpunkt aus kardiovaskulär bedingtem Tod und/oder erster stationärer Aufnahme aufgrund von HI sowie die Rate kardiovaskulär bedingter Tode selbst konnte durch das Kombipräparat signifikant gesenkt werden. Dieser Studie folgend wurde die Therapie bei chronischer $\mathrm{HI}$ in den Leitlinien angepasst und das Kombipräparat erhielt eine Klasse-I-b-Empfehlung für Patienten, die trotz optimaler HI-Medikation (d. h. ACE-Hemmer, Betablocker, und Mineralokortikoidantagonisten in der maximal tolerierbaren Dosis) weiter symptomatisch sind. Die neuen ESC-Guidelines betonen aber, dass eine optimale Standardtherapie (Auftitration!) vorliegen muss, bevor der ARNI eingesetzt werden kann. Laut Leitlinie sollten nur Patienten damit behandelt werden, die die Einschlusskriterien der PARADIGM-HF-Studie erfüllen*.

Nützt der ARNI bei HI mit und ohne Diabetes gleichermaßen?

\section{Was sagt das IQWiG ?}

Das Institut für Qualität und Wirtschaftlichkeit im Gesundheitswesen (IQWiG) hat in einem Addendum (zum Auftrag A 15-60 vom 25.5.2016) die „einzig relevante Studie“ für die Nutzenbewertung von Sacubitril/Valsartan (PARADIGM-HF) anhand von drei Bewertungsgegenständen neu analysiert: Sensitivitätsanalyse zum Einfluss der Run-In-Phase, nachgereichte Analyse zur gesundheitsbezogenen Lebensqualität und zum Gesundheitszustand sowie eine Subgruppenanalyse zum Merkmal „Di-

\footnotetext{
$\mathrm{EF}<35 \%, \mathrm{BNP}>150 \mu \mathrm{g} / \mathrm{ml}$ oder NT-Pro-BNP $>600 \mu \mathrm{g} / \mathrm{ml}$ oder stationärer Aufenthalt wegen $\mathrm{HI}$ im letzten Jahr und BNP $>100 \mu \mathrm{g} / \mathrm{ml}$, NT-Pro-BNP $>400$ $\mu \mathrm{g} / \mathrm{ml}, \mathrm{GFR}>30 \mathrm{ml} / \mathrm{min} / 1,73 \mathrm{~m} 2$, Enalapril sowie Sacubitril/Valsartan werden gut vertragen
}

agnose eines Diabetes mellitus“. Vor Einschluss in die Studie war eine Run-In-Phase vorgeschaltet: Erst mussten die Patienten $2 \mathrm{x}$ $10 \mathrm{mg}$ Enalapril und dann LCZ696 bis 2x $200 \mathrm{mg}$ gut vertragen, nur dann wurden sie aufgenommen und nur für sie gelten die Ergebnisse. Etwa 20\% schieden in der Run-In-Phase aus. Dies kann, wie das IQWiG anmerkte, zur Unterschätzung der Rate unerwünschter Ereignisse führen, und zwar im ARNI-Arm stärker als im Vergleichsarm.

Für die Bewertung des Endpunktes „Gesundheitszustand“ ergibt sich nach Aussage des IQWiG kein Anhalt für einen Zusatznutzen von LCZ696 gegenüber Enalapril.

In der dritten Bewertung wurde die Bedeutung des Subgruppenmerkmals „Diagnose eines Diabetes mellitus“ analysiert. Dies ist wesentlich, da HI bei Diabetikern häufig und prognostisch sehr ungünstig ist. Für den Unterpunkt „Mortalität“ wird vom IQWiG angeführt, dass das Ergebnis von PARADIGM-HF für Diabetiker nicht signifikant ist. Daraus ergibt sich fürs IQWiG „kein Anhalt für einen Zusatznutzen von Sacubitril/ Valsartan gegenüber Enalapril für den Endpunkt Mortalität.“

Müssen HI-Patienten mit Diabetes also anders behandelt werden als solche ohne Diabetes?

\section{Was sagen die europäischen Leitlinien?}

Komorbiditäten werden in den neuen HI-Leitlinien der ESC ausführlich beschrieben. Es wird betont, dass Interventionen, die Morbidität und Mortalität reduzieren, bei eingeschränkter Pumpfunktion unabhängig vom Diabetes gleich wirksam sind [2]. Dies gilt besonders für Betablocker. Eine detaillierte Aussage zu LCZ696 bei Diabetes findet sich nicht.

\section{Was sagt die „einzig relevante" Therapiestudie?}

Die Run-In-Phase von PARADIGM-HF führte möglicherweise zur Unterschätzung der Rate unerwünschter Ereignisse, v.a. mit LCZ696. In der Run-In-Phase hatten 36,2\% Diabetes (753 Pati- 
enten von 2079, die im Run-In ausgeschlossen wurden!). 8442 Patienten wurden in die Studie eingeschlossen, darin 2916 Diabetiker (34,5\%). Der Diabetikeranteil war somit im ausgeschlossenen Kollektiv etwa genauso groß wie im randomisierten. $\mathrm{Be}$ deutet das aber, dass die Studienaussage für Patienten mit und ohne Diabetes mellitus gleichermaßen gilt? In der Originalpublikation von PARADIGM-HF wird ausgeführt, dass der Therapieeffekt für Patienten mit und ohne Diabetes konsistent war [3]. Dies gilt für den primären Endpunkt wie für den kardiovaskulären Tod allein. Beim kardiovaskulären Tod schneidet das Konfidenzintervall bei Diabetikern aber den Neutralitätspunkt.

Der p-Wert für Interaktion für die Subgruppe zeigt mit 0,05 gerade so eine Homogenität und wohl eine Konsistenz mit dem Gesamtergebnis der Studie - also ein Grenzbefund! Die IQWiGAnalyse hat im Gegensatz dazu nicht den Endpunkt „kardiovaskuläre Mortalität“, sondern die Endpunkte „Gesamtmortalität“, „Morbidität“ (HI-Hospitalisierung) und „Lebensqualität“ analysiert. Alles wesentliche Kenngrößen, die unsere Therapie leiten sollten. Für den Endpunkt „Gesamtmortalität“ war das Ergebnis für die Subgruppe der Diabetiker aber nicht homogen!

Ist dies nun für die Entscheidung relevant, LCZ696 bei Diabetespatienten einzusetzen?

\section{Was ist das Behandlungsziel bei Herzinsuffizienz?}

Das Ziel einer jeden Behandlung sollte es sein, die Mortalität zu senken und die Lebensqualität zu erhöhen, letzteres kann unter Umständen für den Patienten entscheidender sein. Die Wirksamkeit einer Therapie kann nur in randomisierten prospektiven Studien geprüft werden. Ein gutes Studiendesign beinhaltet eindeutig bestimmbare medizinische Ereignisse als Endpunkte (z.B. kardiovaskulärer Tod oder noch besser Gesamtmortalität) und möglichst wenige Surrogatmarker wie Blutdruck oder Extrasystolen.

Da die Therapie herzinsuffizienter Patienten in den letzten Jahrzenten deutlich besser wurde, ist es üblich, als primären Endpunkt eine Kombination z.B. aus kardiovaskulärem Tod und/oder erster Hospitalisierung wegen HI einzuführen. Sonst müssten zu große Kollektive untersucht werden, um eine Aussage genügend sicher zu machen. Beachtet werden muss neben der NNT, um ein Ereignis zu verhindern, (number needed to treat - mit 21 bzw. 32 für den primären Endpunkt bzw. CV-Tod sensationell niedrig) auch die NNH (number needed to harm), z.B. Verschlechterung der Nierenfunktion oder symptomatische Hypotonie (LCZ696/ Enalapril 588 vs. 388 Patienten). Gerade Patienten mit niedriger GFR haben ein deutlich erhöhtes Herzinfarkt-, Schlaganfall- und Mortalitätsrisiko.

Die Nutzenbewertung durch das IQWiG aus den Daten der PARADIGM-HF-Studie ergeben einen Hinweis auf beträchtlichen Zusatznutzen für die Kombination Sacubitril/Valsartan gegenüber der zweckmäßigen Vergleichstherapie mit Enalapril im gesamten Studienkollektiv! Sowohl im Hinblick auf Sterblichkeit, Klinikaufenthalte und Lebensqualität überwogen die positiven Effekte den negativen Effekt (nicht schwere Nebenwirkungen) deutlich. Die Aussage von PARADIGM-HF ist hier eineindeutig.

\section{Was sagen Subgruppenanalysen aus?}

Die vordefinierten Subgruppenanalysen schlossen das Merkmal Diabetes ein und waren sowohl im primären Endpunkt als auch im kardiovaskulären Tod homogen. Auch wenn mit $\mathrm{p}=0,05$ ein Grenzbefund vorlag, ist die Studie nicht dafür gepowert den
Einfluss von LCZ696 bei Patienten mit/ohne Diabetes zu analysieren. Sondern sie ist gepowert, um den primären Endpunkt zu bestätigen oder zu widerlegen, und zwar in einer Population mit einem Anteil von ca. 35\% Diabetikern (der Anteil in Deutschland liegt bei 7,2\%, der unentdeckter Diabetiker bei 2,1\%; zusammen 9,3\% (Gesundheitssurvey 2012)). Das Ergebnis der PARADIGM-HF-Studie war also eineindeutig - auch für die Gesamtmortalität in einem Studienkollektiv mit ca. 35\% Diabetikern! Subgruppenanalysen sind mit Vorsicht zu bewerten und in ihrer Wertigkeit nur hypothesengenerierend.

Prof. Roberto Ferrari aus Ferrara hat beim ESC-Kongress in Barcelona $2014 \mathrm{zu}$ Recht formuliert: „Results from subgroups (even if they appear logical and sufficiently large) can only generate hypothesis, which need to be confirmed by specifically designed trials!" Dies gilt unabhängig davon, ob Homogenität zum Studienergebnis vorliegt oder nicht. Und ohne eine Studie mit dem Ziel, diese spezifische Fragestellung zu beantworten, ist eine differenzierte Aussage nicht mit genügender Sicherheit möglich. Dies gilt auch für die Frage zum Nutzen von Sacubitril/Valsartan bei Patienten mit/ohne Einschlusskriterium Diabetes. Sicher mag ein raffiniertes Studiendesign Sinn haben - und für die Professoren McMurray und Packer seinen Wert.

Die Kernaussage der Studie zeigt mit hochsignifikantem pWert den Nutzen der Studienmedikation LCZ696, auch bestätigt durch das IQWiG. Subgruppenanalysen dürfen aber nicht dazu führen, Diabetiker den ARNI vorzuenthalten - dies wäre fatal für die Betroffenen! Ist weiteres Wissen um LCZ696 gefordert, sind weitere Studien nötig. Hier helfen auch die Publikationen von Subgruppenanalysen, z.B. in Abhängigkeit des $\mathrm{HbA}_{1 \mathrm{c}}$ [4] nicht weiter, mögen sie auch noch so attraktiv für Pharmafirmen sein. Die Aussagekraft einer Subgruppenanalyse ist beschränkt [5]. Dies gilt im Übrigen auch für Metaanalysen. Aber auch die Aussagekraft einer einzigen Studie ist beschränkt, egal ob sie die Leitlinien ändert. Patienten sind heterogen und schwer in einem einzigen Studiendesign in ihrer Vielfalt abzubilden.

\section{Was sagt der gesunde Menschenverstand?}

Leitlinien können nur Anhaltspunkte für das medizinische Vorgehen sein. Das Befolgen dieser im Sinne einer „Evidence based Medicine“ kann Leben retten (Zitat Prof. Erland Erdmann, Köln). Aber auch Leitlinien haben Grenzen, und zwar bzgl. der Datenlage (Studiendauer meist nur 3-5 Jahre), der Aussage (Studienpopulation/-design), der Ersteller (medizinische Experten; v.a. wenn „opinion based“ oder „conflict of interest“ vorliegt), in der Anwendung (Abbild des konkret zu behandelnden Patienten in der Leitlinie?) und der Umsetzbarkeit (cost effectivenes). Es ist daher auch nachvollziehbar, dass ein IQWiG eine Maßnahme bewertet. Die Basis kann aber auch hier nur die aktuelle Datenlage sein. Neu bzw. retrospektiv definierte Endpunkte führen dabei nicht zur Steigerung der statistischen Aussagekraft. Die Studie liefert ein Ergebnis für einen vordefinierten primären Endpunkt, einen anderen Endpunkt bewerten zu wollen, forderte eine andere Studie. Auch Subgruppenanalysen liefern keine belastbaren Ergebnisse, nur Hypothesen. Internationale Studien national steuern zu wollen, bleibt ein schwer erreichbares Ziel!

Wir werden mit den vorliegenden Daten leben und arbeiten müssen. Der Patient braucht aber die Therapieentscheidung des Arztes. „To do or not to do?" kann „to be or not to be“ für ihn bedeuten. Der Arzt ist gefordert, herzinsuffiziente Patienten nach dem aktuellen Stand der medizinischen Wissenschaft inkl. Leit- 
linien zu behandeln. Dazu gehört der richtige Einsatz von LCZ696 - für Patienten mit und ohne Diabetes - wenigstens bis wir mehr Daten dazu haben.

Das macht Medizin spannend. Nicht das Dogma des weißhaarigen Professors, sondern die Bewertung des zeitgemäßen, wandelbaren medizinischen Wissens sollte unsere Entscheidungen führen. Und trotz oder gerade wegen aller Fortschritte brauchen wir gesunden Menschenverstand oder „common sense“ aber nicht nur, dies wäre ein Schritt zurück. Die Vielfalt der (medizinischen) Daten, Guidelines, Kommentare und Bewertungen verführt dazu, den Patienten als Mensch zu übersehen. Wenn wir das Bewährte anwenden (ACE-Hemmer, Betablocker und Mineralokortikoidantagonisten in maximal tolerierter Dosis) werden wir den Nutzen einer zusätzlichen Therapie mit LCZ696 erkennen können - nach aktueller Datenlage für Nichtdiabetiker und auch für Diabetiker! Dies sollte common sense sein. - Und: Common sense ist für alle wichtig - er ist die Grundlage der Demokratie.
Literatur:

1. http://eurheartj.oxfordjournals.org/content/37/27/2129

2. Gilbert RE, Krum H. Lancet. 2015 May 23;385(9982):2107-17.

3. McMurray JJ, Packer M, Desai AS et al. N Engl J Med 2014:371(11):993-1004.

4. Kristensen SL et al. Circ Heart Fail. 2016 Jan;9(1). pii: e002560.

5. Schulz KF, Grimes DA. Lancet. 2005 May 7-13;365(9471):1657-61.

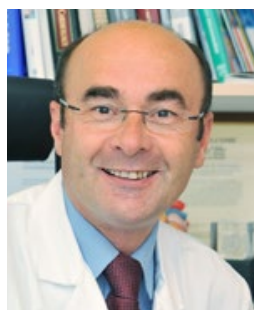

Prof. Dr. med. Robert HG Schwinger

Chefarzt - Med. Klinik II, Kliniken Nordoberpfalz AG, Klinikum Weiden, Akademisches Lehrkrankenhaus der Universität Regensburg Söllnerstr.16, 92637 Weiden i.d. OPf.

Robert.Schwinger@Kliniken-Nordoberpfalz.ag

\section{Jetzt mit pfiffigen Ideen bewerben}

Wie lassen sich Patienten besser versorgen, wie lässt sich die Arztpraxis wirtschaftlich optimieren? Mediziner, die hierauf eine praxistaugliche Antwort gefunden haben, können sich jetzt bewerben und auf den Praxis-Preis 2016 hoffen.

"Lassen Sie Ihre Ideen fliegen“ - unter diesem neuen Motto suchen die Fachverlagsgruppe Springer Medizin, zu der auch „Info Diabetologie“ und die „Ärzte Zeitung“ gehören, und UCB Innere Medizin jetzt Erfolgsrezepte für die Arztpraxis 2016. Etwa solche, die dabei helfen, die Arbeit als Arzt zu vereinfachen, um so Patienten besser versorgen zu können. Abheben können beim Wettbewerb gute Ideen aus allen Bereichen der Arbeit in einer Arztpraxis - es muss dabei auch nicht immer gleich die Welt neu erfunden werden, auch kleine Ideen in der Praxis haben eine Chance. Bürokratie, Ärztemangel, Qualitätsmanagement, Delegation, Compliance, Marketing, Abrechnung, Mitarbeiterführung und der Kampf gegen Volkskrankheiten wie Diabetes und Bluthochdruck - die Themenbandbreite in denen Mediziner innovative Ansätze entwickeln können, um das Unternehmen Arztpraxis zu optimieren, erscheint unerschöpflich. Haben Sie z.B. ein konkretes Konzept entwickelt, das die Wartezeiten für Patienten verkürzt? Setzen Sie spezielle Techniken ein, um das Praxispersonal motiviert und gesund zu halten? Haben sie eine besondere Kooperation mit Kollegen entwickelt, um den Versorgungsdruck zu vermindern? Haben Sie ein besonderes Konzept ausgetüftelt, um die Adhärenz Ihrer Diabetiker zu stärken? Dann bewerben Sie sich bei uns!

\section{Stichtag ist der 30. November}

Ärzte und auch ihre Praxisteams können bis zum 30. November mitmachen und ihre Idee in den Wettbewerb einbringen.
Wer die unabhängige Jury und die InternetNutzer auf aerztezeitung.de mit seinem Projekt, Konzept oder Geistesblitz zur Optimierung der Versorgung beeindruckt, kann wertvolle Preise gewinnen. Die drei Gewinnerteams stellen ihre Konzepte persönlich bei Springer Medizin in Berlin vor.

1. Preis: Ein dreitägiger Ausbildungslehrgang mit Abschluss ,geprüfte Assistenz für Versorgung und Prävention" (Better Care Assistenz). Zudem haben Teilnehmer die Chance auf Buchpreise von Springer Medizin und HCC.

Bewerbungen mit der Beschreibung der eigenen Idee können unkompliziert per Mail an erfolgsrezept@springer.com gesendet werden. „Die Teilnahme an diesem Wettbewerb legen wir anderen Kolleginnen und Kollegen sehr ans Herz. Wir haben uns dafür entschieden, weil wir denken, dass unser Projekt auch für andere Arztpraxen lebbar und sinnvoll ist", zeigen sich die Mediziner Carla Martin \& Michael Pohling aus Flechtorf in Niedersachesn überzeugt. Sie haben 2015 am Wettbewerb teilgenommen und zählten zu den Gewinnern. Durchsetzen konnten sie sich mit ihrem Konzept einer wohnortnahen, hospizähnlichen Palliativversorgung. Geschaffen haben die Ärzte ein ,gemeinde-internes Palliativnetz", das Menschen das Sterben in Wohnortnähe und in der Umgebung von vertrauten Menschen erlaubt.

Marco Hübner

\section{Anmeldung zum Praxis-Preis 2016 \\ Per Internet: aerztezeitung.de/erfolgsrezept \\ PerE-Mail: erfolgsrezept@springer.com \\ Per Post: Ärzte Zeitung Stichwort: Erfolgs-Rezept - Der \\ Praxis-Preis 2016 Postfach 2131, 63243 Neu-Isenburg}

